\title{
Resonance analysis for a space charge dominated beam in a circular lattice
}

\author{
Marco Venturini \\ Stanford Linear Accelerator Center, Stanford University, Stanford, California 94309 \\ Robert L. Gluckstern \\ Physics Department, University of Maryland, College Park, Maryland 20742
}

(Received 22 November 1999; published 27 March 2000)

\begin{abstract}
We use the linearized Vlasov-Poisson equations to study the response of a Kapchinskij-Vladimirskij beam to magnetic multipole errors in a circular lattice. This work extends the calculation of Gluckstern [Proceedings of the Linac Conference, 1970 (Fermilab, Batavia, IL, 1970), p. 811] to the case of nonideal periodic lattices. The smooth approximation is assumed. We determine the resonance conditions as well as the amplitude of the excited collective modes as a function of the error size outside the stopbands. We find that the frequencies associated with lattice resonances are a subset of the beam natural eigenfrequencies. The result is used to study the motion of test particles crossing the boundary of the beam core. Close to resonance the model predicts the emergence of a halo if sufficiently large gradient errors are present. Application is made to the University of Maryland Electron Ring.

PACS numbers: 41.85.-p, 29.20.-c, 29.27.-a, 41.75.-i
\end{abstract}

\section{INTRODUCTION}

In a circular accelerator or storage ring a major limitation to the machine luminosity is caused by the presence of magnetic field errors in the beam optics. The effect of even very small errors can accumulate and lead to instability if the betatron frequencies have resonant values. If there is no coupling between the motion in the horizontal and vertical planes the resonance conditions in terms of the tune in either plane are given by $\nu_{0}=m / n$, where $m$ and $n$ are integers. The number $n$ denotes the order of the resonance. Using a first-order perturbation theory to solve the equation of motions one can associate $n$ to specific components of the multipole spectrum of the lattice errors. Thus, for example, if a tune has values $\nu_{0}=m / 2$ the particle motion is sensitive to quadrupole errors; if $\nu_{0}=m / 3$ the particle motion is sensitive to sextupole errors and so on. Because lattice errors are unavoidable, in designing a circular machine one has to set the tunes to values sufficiently far from resonance. Fortunately, only low order resonances must usually be avoided in order to achieve an acceptable working point. This analysis, based on a single particle approximation of the beam dynamics, provided the theoretical framework in the early days when the importance of space charge as a limiting factor in the performance of synchrotrons was first recognized. In [1] Laslett characterized the effect of space charge as a perturbation producing a shift in the tune

$$
\nu_{0} \rightarrow \nu=\nu_{0}-\Delta \nu .
$$

Because space charge forces act against the external focusing they result in a decrease in the value of the tune. The quantity $\nu$, including the effect of space charge is the "depressed" or "incoherent" tune; $\nu_{0}$ is the "bare" tune. Laslett's problem was to determine the maximum permissible value of $\Delta \nu$.
Space charge forces scale with the inverse square of the beam energy and therefore $\Delta \nu$ decreases during acceleration. Based on the resonance analysis outlined above, a natural criterion to limit $\Delta \nu$ is to impose that in the excursion from the value at injection $\nu_{\text {inj }}=\nu$ to the final value $\nu_{\text {fin }} \simeq \nu_{0}$ the tune does not cross dangerous resonances. Since resonances associated with gradient errors $(n=2)$ are typically the strongest and are separated by half-integers, the above criterion implies

$$
\nu_{\text {fin }}-\nu_{\text {inj }}=\Delta \nu \leq \frac{1}{2} \text {. }
$$

This inequality sets a limit to the maximum beam current that can be accelerated in a synchrotron. Apart from questions regarding the speed with which resonances are crossed (if resonance crossing is sufficiently fast the associated instability may not have enough time to develop), the above criterion turns out to be inaccurate and is, in fact, too restrictive [2-4]. The reason is that the analysis upon which Eq. (1) is based assumes that the depressed tune $\nu$ correctly describes the natural frequency of the system. Although it may be true that $\nu$ represents the linear tune for individual particles, a beam of interacting particles as a whole displays natural frequencies of oscillations that, in general, are different from $\nu$. A correct treatment needs to take into account the collective behavior of the beam. This has long been realized after the work of Smith [5], Sacherer [6], and Gluckstern [7], although occasionally in the literature one still finds arguments backing the criterion (1). (For a detailed discussion of such a misconception, see the paper by Baartman [2].) Unfortunately, an analytical calculation of the resonance conditions for a beam of interacting particles is far more complicated than for the case of noninteracting particles and can be carried out only for a highly simplified modeling of the beam dynamics. Two 
methods have so far been used to tackle the problem. The first is based on the study of the envelope equations (i.e., the equation governing the evolution of the second moments of the beam distribution) and the second on the linearized Vlasov-Poisson equations.

An analysis based on the envelope equations is restricted to the effect of gradient or solenoidal errors. To include the treatment of higher order multipoles one needs to invoke the equations for the moments of higher order, but they are difficult to handle. On the other hand, an approach based on solving the Vlasov-Poisson equations is more general and, in some cases (in the smooth approximation and for beam distributions entailing purely linear space charge forces), the calculation can be carried out analytically. A 1D model of a beam in a uniform focusing was studied using this method by Sacherer [6]; the extension to 2D for an isotropic Kapchinskij-Vladimirskij (KV) [8] beam was carried out by Gluckstern [7], and a further generalization to an unisotropic KV beam (i.e., with unequal emittances and depressed tunes in the horizontal and vertical planes) has recently been presented by Hofmann [9]. This is the method that we will employ in this paper.

Our goal here is to further clarify our understanding of the collective behavior of a beam by placing the emphasis not only on deriving the resonance conditions but also on determining the dependence of the collective modes on the size of the periodic lattice errors that drive them. This will allow us to outline the proper framework to study the motion of test particles and correct some earlier works (see e.g., [10]) that overlooked the space charge perturbation associated with the collective modes.

As in [7], we consider an isotropic KV beam. In our study we find that multipole errors drive collective modes that, in the transverse plane, have the same azimuthal symmetry as the driving multipoles. This implies that only a subset of all the natural eigenfrequencies of the beam can resonate with the lattice errors. Moreover, we find the remarkable result that under certain conditions there is cancellation inside the beam core between the forces associated with a lattice multipole error and those associated with the space charge perturbation excited by that multipole. Specifically, if the lattice multipole error is of order $n$ and has harmonic number $m$, the cancellation takes place if the incoherent tune has values $\nu=m / n$. Thus, for example, if a gradient error distribution has only the harmonic component $m$, the response of the beam is minimum if the tune is $\nu=m / 2$ [which is exactly the value one would try to avoid based on the analysis that led to Eq. (1)]. Finally, we use the calculation of the collective excitations of the beam to study the dynamics of test particles with amplitude of oscillation exceeding the radius of the beam core. The calculation takes into account the forces due to both the gradient errors and the space charge perturbation excited by those errors. We show how these particles under certain conditions can generate a halo surrounding the beam core.
The structure of the paper is as follows. Section II is devoted to the discussion of the model and derivation of the linearized Vlasov-Poisson equations; their solutions are then presented in Sec. III. In Sec. IV we clarify the flaws of a resonance analysis that does not take into account the collective behavior of the beam. Finally, Sec. V contains a discussion on the possibility of halo formation due to resonance crossing. Appendices A and B contain some mathematical details relative to Secs. III and IV, while in Appendix $\mathrm{C}$ we illustrate the mechanism of cancellation between the lattice error and space charge perturbation forces mentioned above using the alternate method of the envelope equations.

\section{THE VLASOV-POISSON EQUATIONS}

The purpose of this section is to derive the linearized Vlasov-Poisson equations obeyed by the beam distribution function. We begin by writing the Hamiltonian relative to our model [11]:

$$
\begin{aligned}
H= & \frac{1}{2}\left(p_{x}^{2}+p_{y}^{2}\right)+\frac{\omega_{0}^{2}}{2}\left(x^{2}+y^{2}\right) \\
& +\frac{q}{m v_{z}^{2} \gamma^{3}} \psi(x, y, z)+\frac{q}{p_{z}} A_{z}(x, y, z) .
\end{aligned}
$$

In this model, a continuous beam of charged particles undergoes uniform linear focusing in both the horizontal and vertical plane and is exposed to the lattice errors described by the $z$ component of the vector potential $A_{z}$. The self-potential $\psi$ takes into account both the electric and magnetic self-forces acting on the particles in the beam. We neglect image charge effects. As for the various parameters appearing in (2), $m$ and $q$ are the mass and charge of the particles, $v_{z}$ and $p_{z}$ are the design velocity and momentum, and $\gamma$ is the relativistic factor. Finally, $\omega_{0}^{2}$ is the smoothened focusing function in the absence of space charge; $\omega_{0}^{2}$ is related to the bare tune $\nu_{0}$ by $\omega_{0}=\nu_{0} / R$, where $R$ is the radius of the machine.

Our goal is to study the behavior of the beam around an equilibrium distribution by investigating the solutions of the Vlasov-Poisson equation associated with the Hamiltonian (2),

$$
\begin{gathered}
\nabla^{2} \psi=-\frac{q}{\epsilon_{0}} \iint d p_{x} d p_{y} f\left(x, p_{x}, y, p_{y} ; z\right), \\
\frac{\partial f}{\partial z}+[f, H]=0,
\end{gathered}
$$

where $[$,$] are the Poisson brackets.$

First, we decompose the Hamiltonian (2) into the sum of an unperturbed $H_{0}$ and perturbed $H_{1}$ term: $H=H_{0}+$ $H_{1}$, where

$$
\begin{aligned}
H_{0}= & \frac{1}{2}\left(p_{x}^{2}+p_{y}^{2}\right)+\frac{\omega_{0}^{2}}{2}\left(x^{2}+y^{2}\right) \\
& +\frac{q}{m v_{z}^{2} \gamma^{3}} \psi_{0}(x, y, z),
\end{aligned}
$$


and

$$
H_{1}=\frac{q}{m v_{z}^{2} \gamma^{3}} \psi_{1}(x, y, z)+\frac{q}{p_{z}} A_{z}(x, y, z),
$$

and where $\psi_{0}$ and $\psi_{1}$ are the unperturbed and perturbed components of the self-potential $\psi=\psi_{0}+\psi_{1}$. The perturbation $H_{1}$ consists of two terms: the perturbed selfpotential $\psi_{1}$ and the external driving force $A_{z}$. Since we are interested in circular lattices, we assume that $A_{z}$ is periodic with period equal to the machine circumference $2 \pi R$. Next, we write the beam distribution function as $f=f_{0}+f_{1}$, where $f_{0}$ is an equilibrium distribution for the unperturbed Hamiltonian $H_{0}$ defined in (4) and $f_{1}$ is an arbitrary, but small, variation of $f$ around $f_{0}$. For $f_{0}$ we chose a KV distribution defined by

$$
f_{0}\left(x, p_{x}, y, p_{y}\right)=\frac{N}{2 \pi^{2} a^{2}} \delta\left(H_{0}-\frac{1}{2} \omega^{2} a^{2}\right),
$$

with

$$
\nabla^{2} \psi_{0}=-\frac{q}{\epsilon_{0}} \iint d p_{x} d p_{y} f_{0}\left(x, p_{x}, y, p_{y}\right) .
$$

Such a choice for $f_{0}$ corresponds to a beam of circular cross section of radius $a$ and uniform density. The function $H_{0}$ is the same as the Hamiltonian (4), $N$ is the linear density, while $\omega^{2}$ is the focusing function including the effect of space charge.

The form (6) for $f_{0}$ is consistent with a $\psi_{0}$ that is quadratic in $x$ and $y$ for $\rho^{2}=x^{2}+y^{2} \leq a^{2}$ as can be verified by solving the Poisson equation (7). In particular, one can see that

$$
\psi_{0}= \begin{cases}-\frac{q}{\epsilon_{0}} \frac{N}{4 \pi a^{2}} \rho^{2}, & \text { for } \rho \leq a, \\ -\frac{q}{\epsilon_{0}} \frac{N}{4 \pi}\left[1+2 \log \left(\frac{\rho}{a}\right)\right], & \text { for } \rho>a .\end{cases}
$$

Therefore, for $\rho \leq a$ the Hamiltonian $H_{0}$ can be rewritten as

$$
H_{0}=\frac{1}{2}\left(p_{x}^{2}+p_{y}^{2}\right)+\frac{\omega^{2}}{2}\left(x^{2}+y^{2}\right)
$$

with

$$
\omega^{2}=\omega_{0}^{2}-\frac{q}{m v_{z}^{2} \gamma^{3}} \frac{q N}{2 \pi \epsilon_{0} a^{2}}=\omega_{0}^{2}-\frac{K}{a^{2}} .
$$

In writing the second equality in (9) we have introduced the generalized perveance $K$ [8],

$$
K=\frac{q^{2} N}{2 \pi \epsilon_{0} m v_{z}^{2} \gamma^{3}} .
$$

We are now ready to derive the equation satisfied by an arbitrary deviation $f_{1}$ of the beam distribution function $f$ from its equilibrium $f_{0}$. The first step is to write the Vlasov equation for $f=f_{0}+f_{1}$,

$$
\frac{\partial f_{0}}{\partial z}+\frac{\partial f_{1}}{\partial z}+\left[f_{0}+f_{1}, H_{0}+H_{1}\right]=0 .
$$

Next, in (11) we make use of the fact that $f_{0}$, as defined in (6), separately satisfies

$$
\frac{\partial f_{0}}{\partial z}+\left[f_{0}, H_{0}\right]=0 .
$$

Therefore, Eq. (11) becomes

$$
\frac{\partial f_{1}}{\partial z}+\left[f_{1}, H_{0}\right]+\left[f_{0}, H_{1}\right]+\left[f_{1}, H_{1}\right]=0 .
$$

In the first-order calculation discussed in this paper we assume that the term $\left[f_{1}, H_{1}\right]$ is negligible compared to the other terms in (13). As a result, the linearized Vlasov equation for $f_{1}$ reads

$$
\frac{\partial f_{1}}{\partial z}+\left[f_{1}, H_{0}\right]=-\left[f_{0}, H_{1}\right] .
$$

This equation is accompanied by the Poisson equation

$$
\nabla^{2} \psi_{1}=-\frac{q}{\epsilon_{0}} \iint d p_{x} d p_{y} f_{1}\left(x, p_{x}, y, p_{y}\right) .
$$

Because of its linearity, the general solution for Eq. (14) can be written as a superposition of the general solution of the homogeneous equation [i.e., Eq. (14) with $A_{z}=0$ ] and a particular solution of the full equation (14).

The case $A_{z}=0$ is the one treated by Gluckstern [7] and leads to an eigenvalue problem for the natural modes of oscillation of the beam. In this paper we focus on the case $A_{z} \neq 0$. To be more specific we now assume the function $A_{z}$, describing the lattice errors, has the form

$$
A_{z}=\sum_{\substack{n \geq 2 \\ m \geq 1}} b_{n, m} e^{-i m \Omega_{0} z} \rho^{n} \cos n \phi,
$$

where $\Omega_{0}$ is the fundamental harmonic of the lattice $\Omega_{0}=1 / R$. The choice (16) corresponds to including all possible magnet multiple errors of order 2 and higher. In particular, $n=2$ corresponds to gradient errors, $n=3$ to sextupole errors, $n=4$ to octupole errors, and so on. In the following, for brevity of notation we will assume that only one multipole error is present with a given harmonic component $\left(\Omega_{e}=m \Omega_{0}\right)$,

$$
A_{z}=A_{z, n} e^{-i \Omega_{e} z} \rho^{n} \cos n \phi .
$$

(Also, we will use the notation $\tilde{A}_{z}=A_{z, n} \rho^{n} \cos n \phi$ for the transverse part of $A_{z}$ ). The most general case can, of course, be recovered by linear superposition.

Equation (14) can be solved as in [7] using the method of the characteristics. The method entails integrating Eq. (14) along the orbits of the unperturbed Hamiltonian $H_{0}$ to obtain the Poisson equation for $\tilde{\psi}_{1}(x, y)$ 
representing the transverse part of $\psi_{1}(x, y, z)$ [i.e., $\left.\psi_{1}(x, y, z)=\tilde{\psi}_{1}(x, y) \exp \left(-i \Omega_{e} z\right)\right]$,

$$
\nabla^{2} \tilde{\psi}_{1}=\frac{2 K}{a^{2}} \frac{1}{e^{-i 2 \pi \lambda}-1}\left[\left.\frac{\delta(\rho-a)}{\omega^{2} a} G\right|_{\xi=0}+\left.\frac{1}{\pi \omega^{2}} \int_{0}^{2 \pi} d \Theta \frac{d \mathcal{G}}{d \xi}\right|_{\xi=a^{2}-\rho^{2}}\right],
$$

where we have defined

$$
\mathcal{G}\left(x, p_{x}, y, p_{y}\right)=\left[\tilde{\psi}_{1}(x, y)+v_{z} \gamma^{2} \tilde{A}_{z}(x, y)\right]\left(e^{-i 2 \pi \lambda}-1\right)+i \lambda \int_{0}^{2 \pi} d \tau\left[\tilde{\psi}_{1}\left(x^{\prime}, y^{\prime}\right)+v_{z} \gamma^{2} \tilde{A}_{z}\left(x^{\prime}, y^{\prime}\right)\right] e^{-i \tau \lambda},
$$

with $x^{\prime}$ and $y^{\prime}$ being the functions introduced in (A1) and $\lambda=\Omega_{e} / \omega$. For the details of the calculation, see Appendix A.

In the right-hand side (RHS) of Eq. (18) we can clearly distinguish a term expressing a density perturbation in the body of the beam and a surface charge term proportional to the $\delta$ function. Equation (18) is therefore best decomposed into an equation for $\rho<a$,

$$
\nabla^{2} \tilde{\psi}_{1}=\left.\frac{2 K}{\pi \omega^{2} a^{2}} \frac{1}{e^{-i 2 \pi \lambda}-1} \int_{0}^{2 \pi} d \Theta \frac{d G}{d \xi}\right|_{\xi=a^{2}-\rho^{2}},
$$

and one for $\rho=a$. The latter is more conveniently written after integration along the variable $\rho$ between a $a_{+}=a+\epsilon$ and $a_{-}=a-\epsilon$, where $\epsilon$ is an infinitesimal quantity,

$$
\begin{aligned}
\int_{a_{-}}^{a_{+}} \nabla^{2} \tilde{\psi}_{1} d \rho= & \frac{2 K}{\omega^{2} a^{3}} \frac{1}{e^{-i 2 \pi \lambda}-1} \\
& \times\left.\int_{a_{-}}^{a_{+}} d \rho \delta(\rho-a) \mathcal{G}\right|_{\xi=0} .
\end{aligned}
$$

Equation (21) expresses the discontinuity of the electric field at the beam boundary due to surface charge accumulation.

After succeeding in writing the equations (20) and (18) satisfied by the perturbation potential $\tilde{\psi}_{1}$, we can now proceed to the next section to determine the solutions.

\section{SOLUTIONS OF THE LINEARIZED EQUATIONS}

In the case of vanishing errors in the lattice $\left(\tilde{A}_{z}=0\right)$, Eq. (18) reduces to the equation first derived in [7] in the study of the natural modes of oscillations of a KV beam in a perfect lattice. Before considering the inhomogeneous case $\tilde{A}_{z} \neq 0$, it is useful to review the results in [7]. Gluckstern found that inside the beam boundary $(\rho \leq a)$, Eqs. (20) and (21) have polynomial solutions of the form

$$
\begin{aligned}
\tilde{\psi}_{1} & =G_{j, n}(\rho, \phi) \\
& =\rho^{n}\left\{\begin{array}{c}
\cos n \phi \\
\sin n \phi
\end{array}\right\}_{2} F_{1}\left(-j, n+j, n+1 ; \rho^{2}\right),
\end{aligned}
$$

where ${ }_{2} F_{1}$ is the hypergeometric function and $j \geq 0$ and $n \geq 0$ are integers. We remind the reader that ${ }_{2} F_{1}$ is defined by

$$
\begin{aligned}
{ }_{2} F_{1}(\alpha, \beta, \gamma ; x)=1 & +\frac{\alpha \beta}{\gamma} \frac{x}{1 !} \\
& +\frac{\alpha(\alpha+1) \beta(\beta+1)}{\gamma(\gamma+1)} \frac{x^{2}}{2 !}+\cdots .
\end{aligned}
$$

The polynomial $G_{j, n}$ represents eigenmodes corresponding to eigenfrequencies $\lambda=\lambda_{j, n}=\Omega_{j, n} / \omega$ that are solutions of the dispersion equation

$$
\begin{aligned}
\int_{0}^{\infty} d s e^{i \lambda s}{ }_{2} F_{1}\left(-j, n+j, n+1 ; \cos ^{2} s\right) \cos ^{n} s \\
=-\frac{1}{i \lambda}\left[\delta_{j 0}+\frac{(-1)^{j} n ! j !}{(n+j-1) !} \frac{\omega^{2}}{\omega_{0}^{2}-\omega^{2}}\right] .
\end{aligned}
$$

The eigenmodes (22) can be distinguished into two classes depending on whether $j=0$ or $j \neq 0$. Clearly, polynomial solutions with $j=0$ are harmonic functions, i.e., $\nabla^{2} G_{j=0, n}=0$, because ${ }_{2} F_{1}\left(0, n, n+1 ; \rho^{2}\right)=1$ and, therefore, from (22) we have $G_{j=0, n}=\rho^{n} \cos n \phi$ or $G_{j=0, n}=\rho^{n} \sin n \phi$.

As a consequence, the density perturbation associated with $G_{j=0, n}$ is confined to the beam boundary $\rho=a$. More in general, each eigenmode $G_{j, n}$ is associated with a beam density perturbation $n_{j, n}(x, y ; z)$ that, according to the RHS of Eq. (18), can be decomposed into the sum of a surface and body term

$$
\begin{aligned}
n_{j, n}(\rho, \phi ; z)=[ & n_{j, n}^{\text {(surf) }}(\phi) \delta(\rho-a) \\
& \left.+n_{j, n}^{(\text {body })}(\rho, \phi) \Theta(a-\rho)\right] e^{-i \Omega_{j, n} z} .
\end{aligned}
$$

Charge conservation entails $\iint n_{j, n}(x, y) d x d y=0$.

It is instructive to write down explicitly the lowest order (in $\rho$ ) modes. For simplicity let us consider only "normal modes," i.e., those modes corresponding to the upper choice of the trigonometric function in Eq. (33). There is one first-order mode $G_{j=0, n=1}=g_{0,1} \rho \cos \phi=g_{01} x$. The corresponding eigenfrequency is $\Omega_{0,1}=\omega_{0}$. This mode has a dipole symmetry.

There are two second-order eigenmodes. The first has a quadrupole symmetry: $G_{j=0, n=2}=g_{2,0} \rho^{2} \cos 2 \phi=$ $g_{2,0}\left(x^{2}-y^{2}\right)$ with eigenfrequency $\Omega_{0,2}^{2}=3 \omega^{2}+\omega_{0}^{2}$ and surface charge density $n_{0,2}^{\text {(surf) }}=8 g_{2,0}\left(x^{2}-y^{2}\right)$. A schematic plot of the total KV-beam density with the $G_{0,2}$ mode excited is shown in Fig. 1.

The other second-order eigenmode has a solenoidal symmetry: $G_{j=1, n=0}=g_{1,0}\left(1-\rho^{2}\right)$ with eigenfrequency 


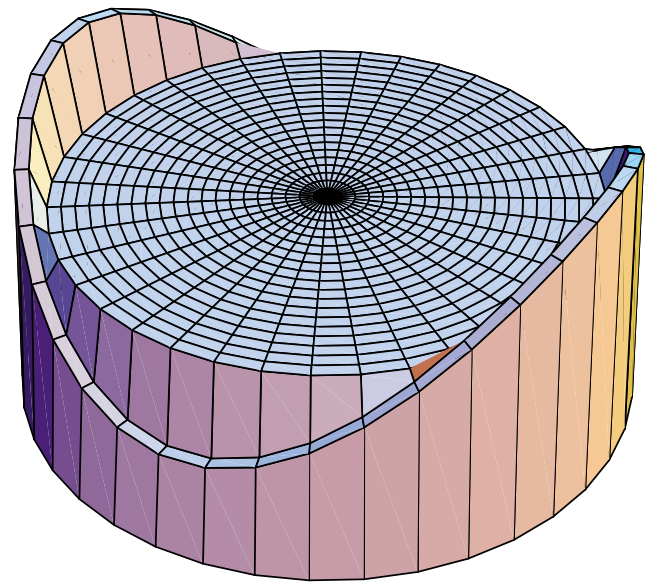

FIG. 1. (Color) KV-beam density in the transverse $x-y$ plane with the quadrupole $G_{0,2}$ mode excited (schematic). This mode can be driven by gradient errors in the lattice and, like all the $G_{0, n}$ modes, results in a perturbation of the charge density confined to the surface of the beam core.

$\Omega_{1,0}^{2}=2 \omega_{0}^{2}+2 \omega^{2}$; this mode has a nonvanishing surface charge density $n_{1,0}^{\text {(surf) }}=4 g_{1,0}\left(x^{2}+y^{2}\right)$ as well as a nonvanishing charge density $n_{1,0}^{\text {(body) }}(x, y)=-4 g_{1,0}$ in the beam core.

In Figs. 2 and 3 we show the schematic plot of a KVbeam density with the modes $G_{0,3}$ and $G_{2,0}$ excited. The mode $G_{0,3}$ has a sextupole symmetry.

The distinction between harmonic and nonharmonic solutions in the homogeneous $\left(\tilde{A}_{z}=0\right)$ case is relevant for determining the solutions of Eqs. (20) and (21) in

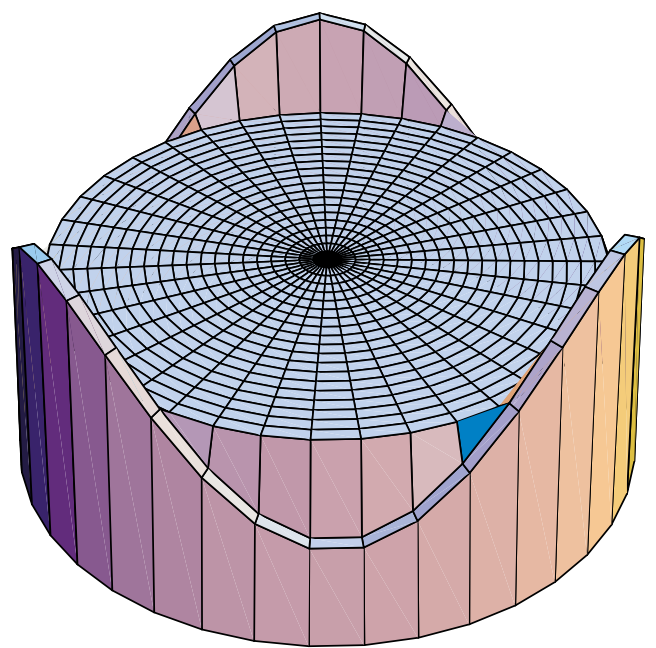

FIG. 2. (Color) KV-beam density with the sextupole $G_{0,3}$ mode excited (schematic). the inhomogeneous case $\left(\tilde{A}_{z} \neq 0\right)$ upon realizing that the lattice error function $\tilde{A}_{z}$ [as defined in the line below Eq. (17)] itself is a harmonic polynomial.

Therefore, for the inhomogeneous case we look for solutions in the form of harmonic functions both inside and outside the beam boundary. In particular, we write the inside-the-beam solution $\rho \leq a$ as

$$
\tilde{\psi}_{1}=\psi_{1, n} \rho^{n} \cos n \phi,
$$

while outside the beam boundary $(\rho>a)$ we have

$$
\tilde{\psi}_{1}=\psi_{1, n} \frac{a^{2 n}}{\rho^{n}} \cos n \phi .
$$

By substituting (24) and (25) into Eq. (21) we find an algebraic equation that determines the amplitude $\psi_{1, n}$ of the perturbed potential as a function of the amplitude $A_{z, n}$ of the driving perturbation.

Incidentally, notice that in this case Eq. (20) is not relevant because it is identically satisfied if $\tilde{\psi}_{1}$ is harmonic (see Appendix B for a proof that indeed $\int_{0}^{2 \pi} d \Theta \frac{d \mathcal{G}}{d \xi}=0$ ).

Upon integration in the RHS of Eq. (21) we have

$$
\int_{a_{-}}^{a_{+}} \nabla^{2} \tilde{\psi}_{1} d \rho=\left.\frac{\partial \tilde{\psi}_{1}}{\partial \rho}\right|_{a_{-}} ^{a^{+}}=-2 n a^{n-1} \psi_{1, n} \cos n \phi .
$$

Moreover [from Eq. (19)], it is

$$
\begin{aligned}
\left.G\right|_{\xi=0}= & {\left[\left(e^{-i \lambda 2 \pi}-1\right)+i \lambda \int_{0}^{2 \pi} d \tau \cos ^{n} \tau e^{-i \tau \lambda}\right] } \\
& \times\left(\psi_{1, n}+v_{z} \gamma^{2} A_{z, n}\right) a^{n} \cos n \phi .
\end{aligned}
$$

After substituting (26) and (27) into Eq. (21) and carrying out the integration of $\rho$ involving the $\delta$ function, we

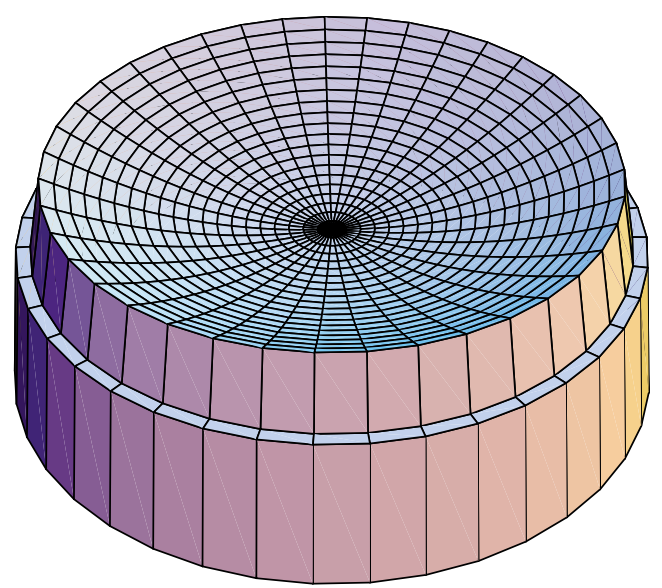

FIG. 3. (Color) KV-beam density with the $G_{2,0}$ mode excited (schematic). This is an example of a mode that cannot be driven by multipole lattice errors: it results in a perturbation of both the core and the surface of the beam core density. 
obtain the following equation for the amplitude $\psi_{1, n}$ :

$$
\begin{aligned}
-\psi_{1, n} n= & \frac{K}{a^{2} \omega^{2}}\left(\psi_{1, n}+v_{z} \gamma^{2} A_{z, n}\right) \\
& \times\left[1+\frac{i \lambda}{e^{-i 2 \pi \lambda}-1} \int_{0}^{2 \pi} d \tau e^{-i \tau \lambda} \cos ^{n} \tau\right],
\end{aligned}
$$

which has the solution $\left(K / a^{2}=\omega_{0}^{2}-\omega^{2}\right)$,

$$
\psi_{1, n}=-v_{z} \gamma^{2} A_{z, n} \frac{B_{n}\left(\Omega_{e} / \omega\right)\left(\omega_{0}^{2}-\omega^{2}\right)}{n \omega^{2}+\left(\omega_{0}^{2}-\omega^{2}\right) B_{n}\left(\Omega_{e} / \omega\right)} .
$$

Here we have defined

$$
B_{n}(\lambda)=1+\frac{i \lambda}{e^{-i 2 \pi \lambda}-1} \int_{0}^{2 \pi} d \tau e^{-i \tau \lambda} \cos ^{n} \tau
$$

The coefficient $B_{n}(\lambda)$ can be calculated using the formula

$$
\beta_{n}(\lambda)=1+\frac{1}{2^{n}} \sum_{k=0}^{\left[\frac{n-1}{2}\right]} \begin{cases}\frac{2 \lambda^{2}}{(n-2 k)^{2}-\lambda^{2}}\left(\begin{array}{l}
n \\
k
\end{array}\right), & \text { for } n=\text { odd } \\
\frac{2 \lambda^{2}}{(n-2 k)^{2}-\lambda^{2}}\left(\begin{array}{l}
n \\
k
\end{array}\right)-\frac{n !}{(n / 2) ! !^{2}}, & \text { for } n=\text { even }\end{cases}
$$

In particular, $B_{2}(\lambda)=2 /\left(4-\lambda^{2}\right)$. The vanishing of with

the denominator $D_{n}\left(\Omega_{e} / \omega\right)=n \omega^{2}+\left(\omega_{0}^{2}-\omega^{2}\right) \times$ $B_{n}\left(\Omega_{e} / \omega\right)$ in Eq. (29) defines the resonant conditions for the system. As expected, the solutions in $\Omega$ of $D_{n}(\Omega / \omega)=0$ coincide with the eigenfrequencies of the $G_{j=0, n}$ modes of Eq. (21); see [7].

In Table I we report the dispersion equations $D_{n}=0$ for $n=2,3,4,5,6$ in terms of the incoherent tune $\nu=$ $\omega R$ and the harmonic number $m\left(\Omega=m \Omega_{0}, \Omega_{0}\right.$ is the fundamental harmonic $\left.\Omega_{0}=1 / R\right)$. Notice that in the limit $\nu \rightarrow \nu_{0}$, the expressions in Table I result in the resonance conditions expected in the absence of space charge. For example, in the presence of gradient errors from $n=2$ and $\nu=\nu_{0}$ we get $\nu=m / 2$; similarly, in the presence of sextupole errors $(n=3)$ we obtain $\nu=m / 3$, etc.

A convenient way to represent the resonance conditions relative to each multipole mode is to draw the curves defined by the equations reported in Table I for $m=$ $1,2,3, \ldots$ on the plane $\left(\nu, \nu_{0}\right)$. Examples of the resulting diagrams are shown in Figs. 4 and 5. Specifically, Fig. 4 displays the resonance curves for quadrupole and sextupole modes, while Fig. 5 reports the resonance curves for all the modes from $n=2$ through $n=5$ in a region of the tune space relevant for the operation of the University of Maryland Electron Ring (UMER) [12].

Finally, we report the density perturbation associated with the potential (24), (25). It is

$$
n_{n}(\rho, \phi)=n_{n}^{\text {surf }}(\phi) \delta(\rho-a),
$$

TABLE I. Resonance conditions.

\begin{tabular}{lc}
\hline \hline$n=2$ (quadrupole) & $\nu_{0}^{2}=-3 \nu^{2}+m^{2}$ \\
$n=3$ (sextupole) & $\nu_{0}^{2}=\nu^{2}-\frac{\left(9 \nu^{2}-m^{2}\right)\left(\nu^{2}-m^{2}\right)}{\left(3 \nu^{2}-m^{2}\right)}$ \\
$n=4$ (octupole) & $\nu_{0}^{2}=\nu^{2}-\frac{\left(16 \nu^{2}-m^{2}\right)\left(4 \nu^{2}-m^{2}\right)}{10 \nu^{2}-m^{2}}$ \\
$n=5$ (decapole) & $\nu_{0}^{2}=\nu^{2}-\frac{\left(25 \nu^{2}-m^{2}\right)\left(9 \nu^{2}-m^{2}\right)\left(\nu^{2}-m^{2}\right)}{45 \nu^{4}-22 m^{2} \nu^{2}+m^{4}}$ \\
$n=6$ (duodecapole) & $\nu_{0}^{2}=\nu^{2}-\frac{\left(36 \nu^{2}-m^{2}\right)\left(16 \nu^{2}-m^{2}\right)\left(4 \nu^{2}-m^{2}\right)}{264 \nu^{4}-40 \nu^{2} m^{2}+m^{4}}$ \\
\hline
\end{tabular}

$$
\begin{aligned}
n_{n}^{\text {surf }}(\phi)= & n_{\mathrm{KV}}\left(\frac{q}{p_{z}} A_{z, n}\right) \frac{a^{n-1} \cos n \phi}{\omega^{2}} \\
& \times \frac{n B_{n}\left(\Omega_{e} / \omega\right)}{n \omega^{2}+\left(\omega_{0}^{2}-\omega^{2}\right) B_{n}\left(\Omega_{e} / \omega\right)},
\end{aligned}
$$

where $n_{\mathrm{KV}}=N /\left(a^{2} \pi\right)$ is the linear density of the unperturbed $\mathrm{KV}$ beam.

We conclude this section by remarking that the appearance of a singular density distribution (Dirac delta function) is an unphysical feature of the solution of our model - a direct consequence of the ansatz (A2). A more physical way to read these results is to interpret the surface density described by a delta function in terms of a displacement of the beam boundary. In an approximation linear in the variation $\delta \rho(\phi)$ of the beam radius, the two pictures are equivalent. Specifically, a KV-beam

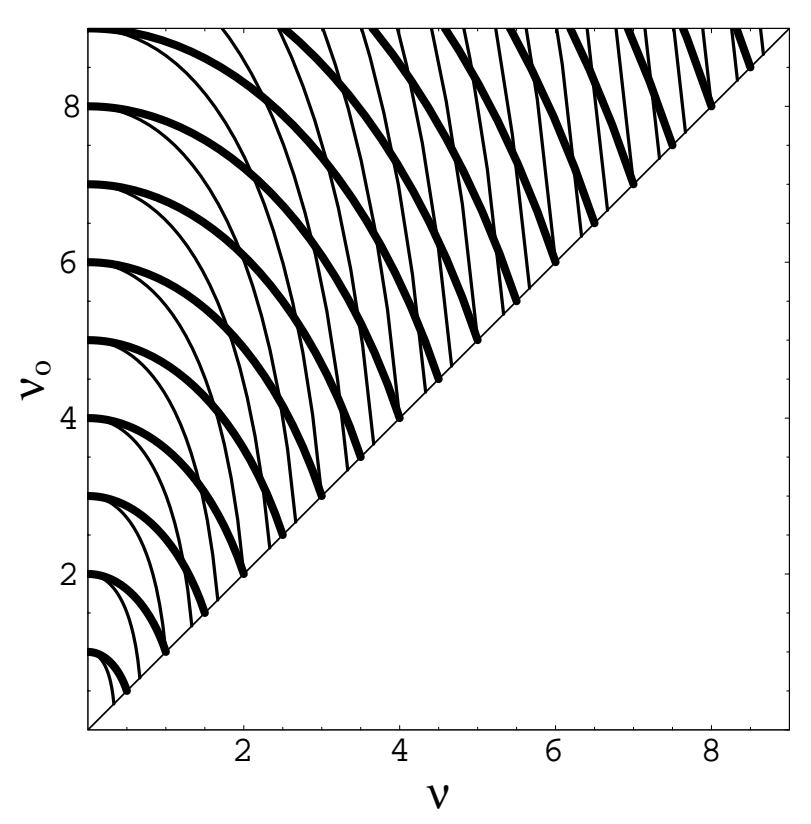

FIG. 4. Tune diagram with the resonance curves for the quadrupole (thicker line) and sextupole (thinner line) beam modes. 


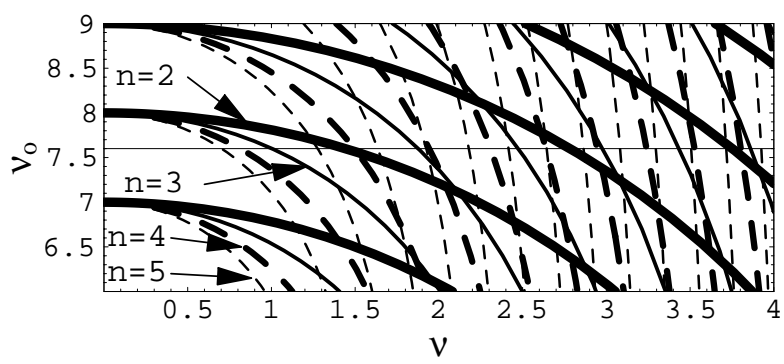

FIG. 5. Tune diagram with the resonance curves for the quadrupole $(n=2)$ through decapole $(n=5)$ beam modes. The horizontal line at $\nu_{0}=7.6$ is the bare tune for the UMER. Notice how the separation between the quadrupole mode resonances becomes substantially larger than $1 / 2$ as the incoherent tune $\nu$ decreases.

equilibrium density distribution with a $n$ pole mode excited can be written as

$$
\begin{aligned}
n(\rho, \phi) & =n_{\mathrm{KV}} \Theta(a-\rho)+n_{n}^{\text {surf }}(\phi) \delta(\rho-a) \\
& =n_{\mathrm{KV}} \Theta[a-\rho-\delta \rho(\phi)]+\mathcal{O}\left[(\delta \rho)^{2}\right],
\end{aligned}
$$

with $\delta \rho(\phi)=n_{n}^{\text {surf }}(\phi) / n_{\mathrm{KV}}$. In particular, for $n=2$, if we write $\left(q / p_{z}\right) 2 A_{z, 2}=\epsilon \omega_{0}^{2}$ (so that $\epsilon$ has the meaning of relative gradient error), we have

$$
\delta \rho(\phi)=\epsilon \nu_{0}^{2} \frac{a \cos 2 \phi}{3 \nu^{2}+\nu_{0}^{2}-m^{2}} .
$$

\section{MOTION OF A TEST PARTICLE WITHIN THE BEAM CORE}

In the previous section we showed that a KV beam responds resonantly to multipole errors in a periodic lattice if the harmonic content of a multipole error distribution matches the natural frequency of the mode excited by that multipole. We saw that, in general, these eigenfrequencies do not equal rational values of the incoherent tune. This appears counterintuitive if one tries to understand the effect of multipole errors in the presence of space charge in a framework, modeled after the familiar single-particle theory, in which the only effect of the space charge is to depress the tune (see [10] for an example of this approach). In that framework, the motion of an individual particle is described by the unperturbed Hamiltonian of Eq. (4) [or Eq. (8)] plus a perturbation consisting of the lattice multipole errors as in Eq. (16); i.e.,

$$
H=\frac{1}{2}\left(p_{x}^{2}+p_{y}^{2}\right)+\frac{\omega^{2}}{2}\left(x^{2}+y^{2}\right)+\frac{q}{p_{z}} A_{z} .
$$

Using such a model one would actually find the resonances located at $\nu=m / n$, where $n$ is the order of the multipole error. In light of the analysis carried out in the previous section, it should now be apparent why this is incorrect: it does not include as a perturbation the term proportional to $\psi_{1}$ in Eq. (5), which describes the redistribution of space charge due to the lattice error $A_{z}$. Because the term with $\psi_{1}$ is of the same order as the one with $A_{z}$, it appears plausible that its presence can alter the results of the resonance analysis. Indeed, this is the case.

The correct Hamiltonian for a single particle whose motion is confined within the beam boundary $(\rho \leq a)$ in a first-order approximation is

$$
\begin{aligned}
H= & \frac{1}{2}\left(p_{x}^{2}+p_{y}^{2}\right)+\frac{\omega^{2}}{2}\left(x^{2}+y^{2}\right) \\
& +\frac{q}{m v_{z}^{2} \gamma^{3}} \psi_{1}+\frac{q}{p_{z}} A_{z} .
\end{aligned}
$$

apub zl7102As shown in the previous section, for a given $A_{z}$ the expression for $\psi_{1}$ can be calculated by solving the Vlasov-Poisson equations. It is a remarkable feature of the KV beam model that if $A_{z}$ represents a pure multipole as defined in (17) (of order $n$ and with harmonic number $m, \Omega_{e}=m \Omega_{0}$ ), then the strength of the total perturbation $H_{1}=\frac{q}{m v^{2} \gamma^{3}} \psi_{1}+\frac{q}{p_{z}} A_{z}$ vanishes exactly for $\nu=n / m$ if $\rho \leq a$. This can be seen easily: $H_{1}$ is proportional to $\psi_{1, n}+v_{z} \gamma^{2} A_{z, n}$ with $\psi_{1, n}$ given by (29). Therefore, we have

$$
\psi_{1, n}+v_{z} \gamma^{2} A_{z, n}=v_{z} \gamma^{2} \frac{n \omega^{2}}{n \omega^{2}+\left(\omega_{0}^{2}-\omega^{2}\right) B_{n}\left(\Omega_{e} / \omega\right)} ;
$$

then, by inspecting formula (31), notice that $B_{n}\left(\Omega_{e} / \omega\right) \rightarrow \infty$ (and therefore $H_{1} \rightarrow 0$ ) if $\lambda \equiv \Omega_{e} / \omega \equiv$ $m / \nu= \pm(n-2 k)$ with $k=0,1, \ldots,[n / 2]$. In particular $(k=0)$, this is true if it is $\nu=m / n$. Thus, for example, the effect of gradient errors in the lattice is exactly compensated by the space charge perturbation induced by those errors if $\nu$ is a half-integer $\nu=m / 2$. We conclude that in the framework of our first-order model, if a particle remains inside the core of a $\mathrm{KV}$ beam, a rational value of the incoherent tune does not carry any particular meaning. We should add, however, that this conclusion does not apply if one considers the motion of a test particle that may fall outside the beam core where the space charge forces cannot compensate for the lattice errors. In that case, as we shall see in the next section, a rational value of the (incoherent) tune has again a resonant meaning and can be associated with the emergence of a halolike structure.

\section{EFFECT OF GRADIENT ERRORS ON THE MOTION OF A TEST PARTICLE}

Here we address more in depth the study of the motion of a single test particle under the influence of a KV beam perturbed by quadrupole errors in the lattice. We consider the motion of a test particle that, while undergoing betatron oscillations, falls outside the beam core radius. The goal is to study the amplitude of the excursions 
outside the beam core, where the particle experiences the nonlinearities due to space charge. This study can cast some light on understanding the limitations of the beam model considered in this paper, as well as the mechanism of beam blowup close to a resonance that can take place, for example, during resonance crossing in the operation of a synchrotron.

For simplicity we restrict ourselves to a reduced 1D system corresponding to the motion of a test particle in the horizontal plane. Specifically, the 1D Hamiltonian we want to study is

$$
H=\frac{1}{2}\left(p_{x}^{2}+\omega^{2} x^{2}\right)+H_{a}+H_{b} \cos \Omega z,
$$

where

$$
H_{a}=\frac{K}{a^{2}}\left(\frac{x^{2}}{2}-a \log \frac{|x|}{a}-\frac{a^{2}}{2}\right) \Theta(|x|-a)
$$

describes the space charge nonlinear forces the particle experiences outside the beam core $(|x|>a)$, and

$$
\begin{aligned}
H_{b}= & \frac{\epsilon}{2} \omega_{0}^{2} x^{2}+\psi_{10}\left(x^{2}-a^{2}\right) \Theta(a-|x|) \\
& +\psi_{10} a^{2}\left(\frac{a^{2}}{x^{2}}-1\right) \Theta(|x|-a) .
\end{aligned}
$$

In the expression above, $\epsilon$ is the relative strength of the gradient errors; these errors excite a quadrupole mode in the beam with amplitude [second and third terms in the RHS of (41)],

$$
\psi_{10}=-\frac{\epsilon}{2} \nu_{0}^{2} \frac{1}{R^{2}} \frac{\nu_{0}^{2}-\nu^{2}}{3 \nu^{2}+\nu_{0}^{2}-m^{2}},
$$

as determined from (29) with $\psi_{10}=\left(q / m v_{z}^{2} \gamma^{3}\right) \psi_{1,2}$. Again, we are assuming that only one harmonic component of the multipole error is present, i.e., $\Omega=m / R$ (again, $\nu=\omega R$ is the incoherent tune).

We are interested in the situation in which both the following conditions are met: the beam is close to a quadrupole mode resonance, i.e., the denominator in (42) is small and $\nu$ is close to $m / n$,

$$
3 \nu^{2}+\nu_{0}^{2}-m^{2} \simeq 0, \quad \nu \simeq m / n .
$$

We will refer to the first condition as a "beam resonance" and to the second as a "particle resonance." The second condition allows us to apply the method of phase averaging to the study of the test particle orbit. To this end it is convenient to rewrite the Hamiltonian (39) using the action-angle variables $(x=\sqrt{2 J / \omega} \sin \phi$ and $\left.p_{x}=\sqrt{2 J \omega} \cos \phi\right)$,

$$
H=\omega J+H_{a}+H_{b} \cos \Omega z,
$$

where now $H_{a}$ and $H_{b}$ have to be thought of as functions of $J$ and $\phi$. Next, we average over all the fast terms leaving

$$
\begin{aligned}
\langle H\rangle= & \omega J+\left\langle H_{a}\right\rangle+\frac{1}{2} \beta_{n} \cos (n \phi-\Omega z) \\
& +\frac{1}{2} \gamma_{n} \cos (n \phi-\Omega z),
\end{aligned}
$$

where $\beta_{n}$ and $\gamma_{n}$ are the $n$ coefficients of the Fourier series representation of the term $H_{b}$; i.e.,

$$
\begin{aligned}
& \beta_{n}=\frac{1}{\pi} \int_{0}^{2 \pi} d \phi H_{b}(J, \phi) \cos n \phi, \\
& \gamma_{n}=\frac{1}{\pi} \int_{0}^{2 \pi} d \phi H_{b}(J, \phi) \sin n \phi,
\end{aligned}
$$

and

$$
\left\langle H_{a}\right\rangle=\frac{1}{2 \pi} \int_{0}^{2 \pi} H_{a}(J, \phi) d \phi .
$$

In our model, the $\gamma_{n}$ vanish. A further canonical transformation,

$$
J \rightarrow J^{\prime}=J, \quad \phi \rightarrow \phi^{\prime}=\phi-z \frac{\Omega}{2},
$$

generated by the function $F_{2}=J^{\prime}(\phi-z \Omega / n)$, is then introduced to produce the invariant $H^{\prime}$,

$$
\begin{aligned}
H^{\prime} & =H+\frac{\partial}{\partial z} F_{2} \\
& =\left(\omega-\frac{\Omega}{n}\right) J+\left\langle H_{a}\right\rangle+\frac{1}{2} \beta_{n} \cos n \phi^{\prime} .
\end{aligned}
$$

The invariant (49) completely determines the orbits of the averaged Hamiltonian. Before considering explicitly the case $n=2$, it is convenient to introduce the normalized amplitude of oscillation $\sigma=\sqrt{2 J /\left(\omega a^{2}\right)}$ and the angle $\phi_{1}=\arcsin (1 / \sigma)$, defined for $\sigma \geq 1$. The value $\sigma=1$ corresponds to the beam core radius.

For $\sigma \leq 1$ we have $\left\langle H_{a}\right\rangle=0$, and for $\sigma>1$,

$$
\left\langle H_{a}\right\rangle=\frac{a^{2}}{R^{2}}\left(\nu_{0}^{2}-\nu^{2}\right)\left[\left(2 \phi_{1}-\pi\right)\left(\frac{1}{2}-\frac{1}{4} \sigma^{2}+\log \sigma\right)+\frac{1}{4} \sigma^{2} \sin 2 \phi_{1}+2 \int_{1}^{\sigma} \frac{\log z}{z \sqrt{z^{2}-1}} d z\right] .
$$

Moreover, for $\sigma \leq 1$ it is

$$
\beta_{2}=-\frac{\epsilon}{4} \sigma^{2} \nu_{0}^{2} \frac{a^{2}}{R^{2}} \frac{4 \nu^{2}-m^{2}}{3 \nu^{2}+\nu_{0}^{2}-m^{2}},
$$

while for $\sigma>1$,

$$
\beta_{2}=\frac{\psi_{10} a^{2}}{\pi}\left[\frac{\sin 4 \phi_{1}}{4 \sin ^{2} \phi_{1}}-\phi_{1}+4 \sin 2 \phi_{1}-4 \sin ^{2} \phi_{1}\left(\pi-2 \phi_{1}\right)\right]-\frac{\epsilon}{4} \nu_{0}^{2} \frac{a^{2}}{R^{2}} \sigma^{2} .
$$




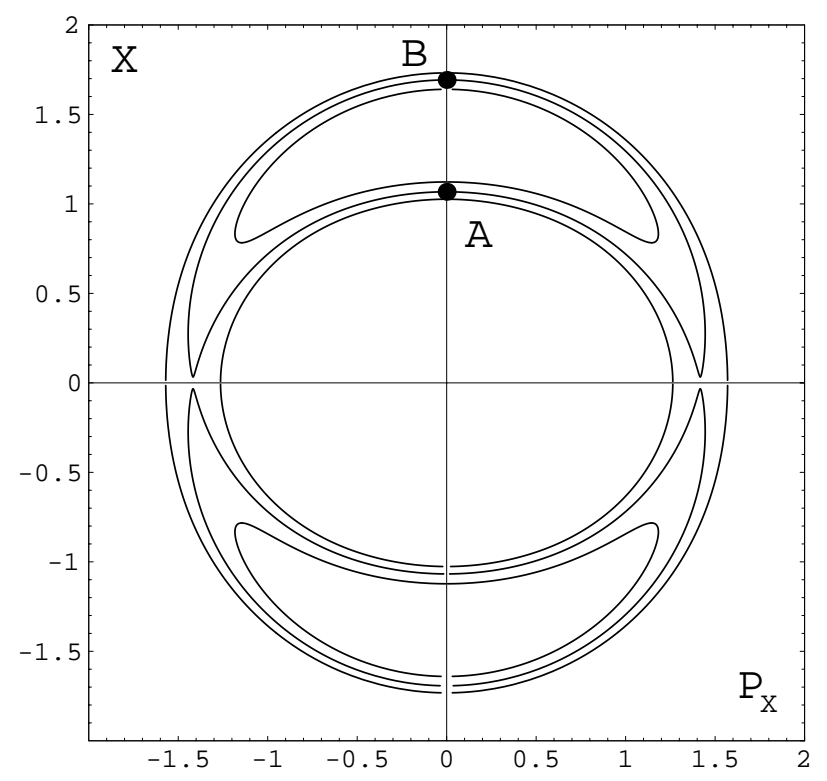

FIG. 6. Phase space orbits for a test particle close to a second-order resonance with the beam close to a quadrupole mode beam resonance; $\nu=3.88, \epsilon=4 \times 10^{-3}$. We use the dimensionless variables $X=\sigma \sin \phi^{\prime}$ and $P_{X}=\sigma \cos \phi^{\prime}$; the unperturbed beam core radius is $\sigma=1$ (see the text for the definition of $\sigma$ and $\phi^{\prime}$ ).

The expressions above can be used to determine the averaged orbits in the phase space. An example is given in Fig. 6. As we can see, the condition $\nu \simeq m / 2$ results in the familiar appearance of two resonance islands. The parameters are $m=8, \nu=3.88 \simeq 8 / 2$, and $\nu_{0}=4.25$. For these values of $\nu_{0}$ and $m$, the quadrupole beam resonance takes place at $\nu \simeq 3.91$.

The location and extent of the resonance structure depend on the strength of the gradient errors and vicinity to the quadrupole mode beam resonance. In Fig. 7 we present a systematic study of the location of the minimum

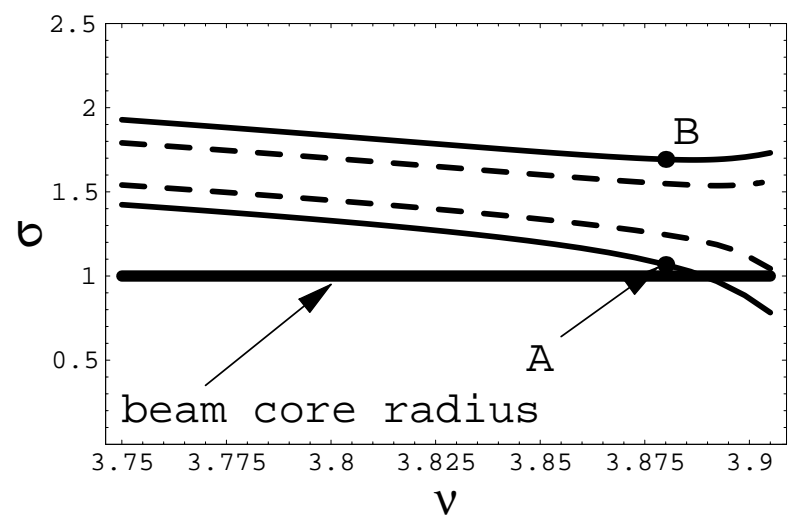

FIG. 7. Maximum and minimum distance of the resonance separatrix from the origin of the phase space for $\epsilon=4 \times 10^{-3}$ (solid lines) and $\epsilon=10^{-3}$ (dashed line) in units of the unperturbed beam core radius. The points $A$ and $B$ are the same as in Fig. 6. and maximum distance of the resonance separatrix from the origin of the phase space as a function of the incoherent tune. Two cases are shown: $\epsilon=4 \times 10^{-3}$ (solid lines) and $\epsilon=10^{-3}$ (dashed lines). Notice that the resonance islands approach the beam boundary $(\sigma=1)$ as the beam gets close to the quadrupole beam resonance. For example, for $\epsilon=4 \times 10^{-3}$ at $\nu \simeq 3.885$ the orbit of a particle locked in the resonance would merge with the beam core. Clearly, this signals a breakdown of the model because such a dynamic is inconsistent with a KV beam distribution. This inconsistency may be interpreted in terms of the crossing of the resonance stopband. At this point a more accurate calculation would require going beyond our first-order approximation.

Higher order particle resonances (i.e., $\nu \simeq m / n$ with $n>2$ ) are also possible although, as one could expect, they are weaker than the $n=2$ resonance. The reason is that for $\sigma \leq 1$ the driving term of the resonance averages to zero (i.e., $\beta_{n}=0$ for $n>2$ ). As an example, we show the phase-space orbits for the case with $m=9$, $\nu=2.2 \simeq 9 / 4$, and $\nu_{0}=8.1124$ (see Fig. 8). For this case, $\left\langle H_{a}\right\rangle$ is the same as in (50), while for $\sigma \leq 1$, $\beta_{4}=0$, and for $\sigma>1$ we have

$$
\begin{aligned}
\beta_{4}= & \frac{\psi_{10} a^{2}}{\pi} \\
\times & {\left[\frac{3 \sin 4 \phi_{1}-3 \sin 2 \phi_{1}-\sin 6 \phi_{1}}{6 \sin ^{2} \phi_{1}}\right.} \\
& \left.\quad+4 \sin ^{2} \phi_{1}\left(\cot \phi_{1}+2 \sin 2 \phi_{1}+4 \phi_{1}-2 \pi\right)\right] .
\end{aligned}
$$

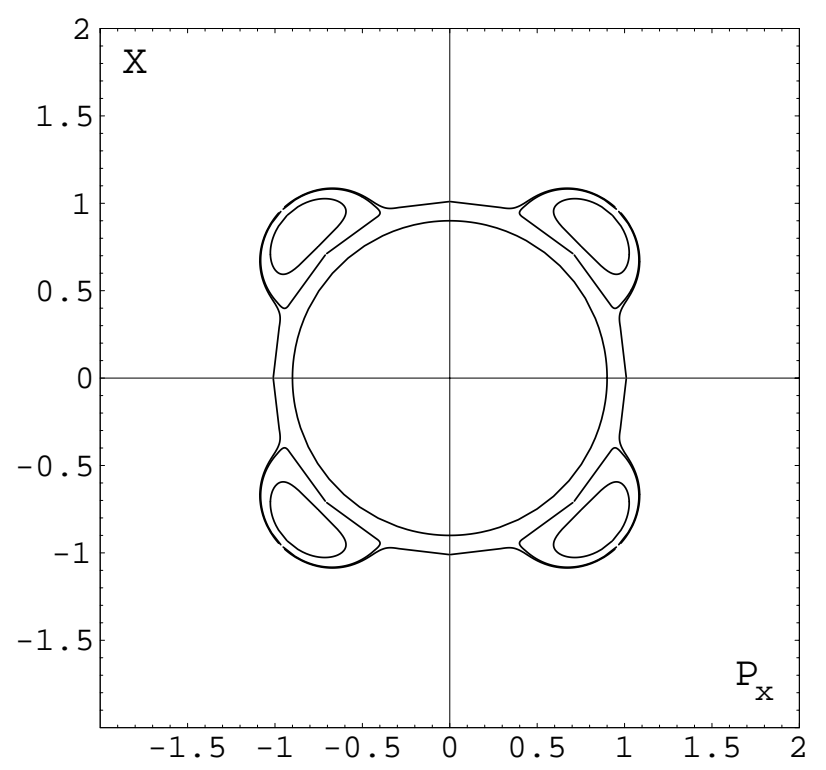

FIG. 8. Phase space orbits for a test particle close to a fourthorder resonance with the beam close to a quadrupole mode beam resonance; $\nu=2.2, \epsilon=4 \times 10^{-3}$ ( $X$ and $P_{X}$ are the same variables as in Fig. 6). 


\section{DISCUSSION}

First, a comment on the implications of having assumed the smooth approximation for the external focusing. An interesting question is the extent to which the results of our resonance analysis can be applied to an alternate gradient (AG) lattice. Although the ultimate answer can be given only by comparisons with numerical simulations, there is already some evidence in the literature that can be used to address that question. In particular, in [13] one finds that an AG lattice does not appear to change significantly the eigenfrequencies of a $\mathrm{KV}$ as calculated in the smooth approximation (unless resonances excited by the AG flutter become significant driving rapid instabilities). In [14] a similar result was found, where instead of the AG flutter a mismatch in a KV beam in a solenoidal smooth channel was considered.

Finally, some remarks on the model presented in Section V. Our calculations show that close to a quadrupole mode beam resonance (i.e., when the response of the beam to gradient errors is the largest) a test particle that happens to fall outside the beam core may get locked into a resonance if the incoherent tune $\nu$ is close to a rational number $\nu=m / n$. As a result, the particle can experience relatively large excursion outside the beam core. The effect is largest if $n=2$. This is the 2:1 parametric resonance that was recognized in [15] as a mechanism for halo formation in linacs. There the driving term for the resonance was indicated in a beam mismatch. Our model suggests that a halo could result in the process of resonance crossing if the gradient errors are sufficiently large. We stress that both conditions (43), i.e., proximity to beam and particle resonances, have to be met in order for a halo to emerge. This poses some restrictions. One can easily see that for $n=2$ this is the case only if $\nu \simeq \nu_{0} \simeq m / 2$, i.e., only if space charge forces are moderate. On the other hand, in a highly space charge dominated regime (in which $\nu / \nu_{0}$ is significantly less than one), both conditions (43) can be satisfied only for $n>2$. Therefore one would expect a less extended halo in that case.

\section{ACKNOWLEDGMENTS}

We are grateful to A. Fedotov, R. Kishek, S. Bernal, and M. Reiser for helpful discussions and useful comments. This work was supported by the U.S. Department of Energy.

\section{APPENDIX A}

This Appendix contains a detailed derivation of Eq. (18) from Eqs. (14) and (15). The first step is to integrate
Eq. (14) along the orbits of the unperturbed Hamiltonian $H_{0}$. To this end we need the map generated by $H_{0}$ of $\left(x^{\prime}, p_{x}^{\prime}, y^{\prime}, p_{y}^{\prime}\right)$ at $z^{\prime}$ in terms of $\left(x, p_{x}, y, p_{y}\right)$ at $z$ :

$$
\begin{aligned}
x^{\prime} & =x \cos \left[\omega\left(z^{\prime}-z\right)\right]+\frac{p_{x}}{\omega} \sin \left[\omega\left(z^{\prime}-z\right)\right], \\
p_{x}^{\prime} & =-x \omega \sin \left[\omega\left(z^{\prime}-z\right)\right]+p_{x} \cos \left[\omega\left(z^{\prime}-z\right)\right], \\
y^{\prime} & =y \cos \left[\omega\left(z^{\prime}-z\right)\right]+\frac{p_{y}}{\omega} \sin \left[\omega\left(z^{\prime}-z\right)\right], \\
p_{y}^{\prime} & =-y \omega \sin \left[\omega\left(z^{\prime}-z\right)\right]+p_{y} \cos \left[\omega\left(z^{\prime}-z\right)\right] .
\end{aligned}
$$

The functions $f_{1}$ and $\psi_{1}$ have an explicit and implicit dependence on the independent variable $z$. The implicit dependence is through the dynamical variables. We make the ansatz that the explicit $z$ dependence can be factored out and write

$$
f_{1}=\tilde{f}_{1}(s) e^{-i \Omega_{e} z}, \quad \psi_{1}=\tilde{\psi}_{1}(s) e^{-i \Omega_{e} z},
$$

where $s$ is the angular variable $s=\omega z$ and $\Omega_{e}$ is the same frequency as in the driving term (17). From (A2) and following [13] we compute the total derivative of $f_{1}$ with respect to $z$,

$$
\frac{d f_{1}}{d z}=\omega \frac{d \tilde{f}_{1}}{d s} e^{-i \Omega_{e} z}-i \Omega_{e} \tilde{f}_{1}(s) e^{-i \Omega_{e} z},
$$

and then integrate over a period of the unperturbed motion

$$
\int_{s}^{s+2 \pi} d s^{\prime} \frac{d f_{1}}{d z}=\omega e^{-i \lambda s}\left[e^{-i 2 \pi \lambda}-1\right] \tilde{f}_{1}(s),
$$

where we have introduced the notation $\lambda=\Omega_{e} / \omega$. Therefore, from (A4) we obtain

$$
\tilde{f}_{1}(s)=\frac{1}{\omega} \frac{e^{i \lambda s}}{e^{-i 2 \pi \lambda}-1} \int_{s}^{s+2 \pi} \frac{d f_{1}}{d z} d s^{\prime} .
$$

Next, we use the linearized Vlasov equation (14) in (A5) with the understanding that

$$
\frac{d f_{1}}{d z} \equiv \frac{\partial f_{1}}{\partial z}+\left[f_{1}, H_{0}\right]
$$

represents the derivative of $f_{1}$ along the orbits of the unperturbed motion, and write

$$
\tilde{f}_{1}(s)=-\frac{1}{\omega} \frac{e^{i \lambda s}}{e^{-i 2 \pi \lambda}-1} \int_{s}^{s+L}\left[f_{0}, H_{1}\right] d s^{\prime} .
$$

Under the integral in Eq. (A7) it is understood that $f_{0}$ and $H_{1}$ are to be thought of as functions of the "primed" variables defined in (A1); e.g.,

$$
H_{1}=H_{1}\left[x^{\prime}\left(x, p_{x}, s\right), y^{\prime}\left(x, p_{x}, s\right), z\right] .
$$

From the definitions (5) and (6) we have

$$
\begin{aligned}
{\left[f_{0}, H_{1}\right] } & =-\frac{\partial f_{0}}{\partial p_{x}^{\prime}} \frac{\partial H_{1}}{\partial x^{\prime}}-\frac{\partial f_{0}}{\partial p_{y}^{\prime}} \frac{\partial H_{1}}{\partial y^{\prime}} \\
& =-\frac{N}{2 \pi^{2} a^{2}} \delta^{\prime}\left(H_{0}-\frac{1}{2} \omega^{2} a^{2}\right)\left[\frac{q}{m v_{z}^{2} \gamma^{3}}\left(p_{x}^{\prime} \frac{\partial \tilde{\psi}_{1}}{\partial x^{\prime}}+p_{y}^{\prime} \frac{\partial \tilde{\psi}_{1}}{\partial y^{\prime}}\right)+\frac{q}{p_{z}}\left(p_{x}^{\prime} \frac{\partial \tilde{A}_{z}}{\partial x^{\prime}}+p_{y}^{\prime} \frac{\partial \tilde{A}_{z}}{\partial y^{\prime}}\right)\right] e^{-i \Omega_{e} z} .
\end{aligned}
$$


Let us define the function

$$
\mathcal{G}\left(x, p_{x}, y, p_{y}\right)=\int_{s}^{s+2 \pi} d s^{\prime}\left[\left(p_{x}^{\prime} \frac{\partial \tilde{\psi}_{1}}{\partial x^{\prime}}+p_{y}^{\prime} \frac{\partial \tilde{\psi}_{1}}{\partial y^{\prime}}\right)+v_{z} \gamma^{2}\left(p_{x}^{\prime} \frac{\partial \tilde{A}_{z}}{\partial x^{\prime}}+p_{y}^{\prime} \frac{\partial \tilde{A}_{z}}{\partial y^{\prime}}\right)\right] e^{-i\left(s^{\prime}-s\right) \lambda},
$$

so that Eq. (A7) can be written as

$$
\tilde{f}_{1}(s)=\frac{N}{2 \pi^{2} a^{2}} \frac{q}{m v_{z}^{2} \gamma^{3}} \frac{1}{\omega} \frac{1}{e^{-i 2 \pi \lambda}-1} \delta^{\prime}\left(H_{0}-\frac{1}{2} \omega^{2} a^{2}\right) \mathcal{G}\left(x, p_{x}, y, p_{y}\right) .
$$

After introducing the change of variable $s^{\prime}-s=\tau$ in Eq. (A9) and recognizing [see Eq. (A1)] that

$$
\begin{aligned}
& \frac{d \tilde{\psi}_{1}}{d \tau}=\frac{\partial \tilde{\psi}_{1}}{\partial x^{\prime}} \frac{d x^{\prime}}{d \tau}+\frac{\partial \tilde{\psi}_{1}}{\partial y^{\prime}} \frac{d y^{\prime}}{d \tau}=\frac{1}{\omega}\left(\frac{\partial \tilde{\psi}}{\partial x^{\prime}} p_{x}^{\prime}+\frac{\partial \tilde{\psi}_{1}}{\partial y^{\prime}} p_{y}^{\prime}\right), \\
& \frac{d \tilde{A}_{z}}{d \tau}=\frac{\partial \tilde{A}_{z}}{\partial x^{\prime}} \frac{d x^{\prime}}{d \tau}+\frac{\partial \tilde{A}_{z}}{\partial y^{\prime}} \frac{d y^{\prime}}{d \tau}=\frac{1}{\omega}\left(\frac{\partial \tilde{A}_{z}}{\partial x^{\prime}} p_{x}^{\prime}+\frac{\partial \tilde{A}_{z}}{\partial y^{\prime}} p_{y}^{\prime}\right),
\end{aligned}
$$

we can write the function $G$ defined in Eq. (A9) as

$$
\mathcal{G}\left(x, p_{x}, y, p_{y}\right)=\int_{0}^{2 \pi} d \tau\left[\frac{d \tilde{\psi}_{1}}{d \tau}+v_{z} \gamma^{2} \frac{d \tilde{A}_{z}}{d \tau}\right] e^{-i \tau \lambda} .
$$

An integration by parts and the observation that at $\tau=2 \pi$ we have $\left(x^{\prime}, p_{x}^{\prime}, y^{\prime}, p_{y}^{\prime}\right)=\left(x, p_{x}, y, p_{y}\right)$ finally lead us to

$$
\mathcal{G}\left(x, p_{x}, y, p_{y}\right)=\left[\tilde{\psi}_{1}(x, y)+v_{z} \gamma^{2} \tilde{A}_{z}(x, y)\right]\left(e^{-i 2 \pi \lambda}-1\right)+i \lambda \int_{0}^{2 \pi} d \tau\left[\tilde{\psi}_{1}\left(x^{\prime}, y^{\prime}\right)+v_{z} \gamma^{2} \tilde{A}_{z}\left(x^{\prime}, y^{\prime}\right)\right] e^{-i \tau \lambda} \text {. }
$$

The function $\tilde{f}_{1}(s)$ in (A10) with $G$ given by (A13) can now be inserted into the RHS of the Poisson equation (15),

$$
\nabla^{2} \psi_{1}=-\frac{2 K}{\pi a^{2}} \frac{1}{e^{-i 2 \pi \lambda}-1} \iint d p_{x} d p_{y} \delta^{\prime}\left(H_{0}-\frac{1}{2} \omega^{2} a^{2}\right) \mathcal{G}\left(x, p_{x}, y, p_{y}\right) .
$$

Finally, we integrate over the transverse momenta with $p_{x}=\omega \sqrt{\xi} \cos \Theta, p_{y}=\omega \sqrt{\xi} \sin \Theta$,

$$
\begin{aligned}
\nabla^{2} \psi_{1} & =-\frac{K}{\pi a^{2}} \frac{1}{e^{-i 2 \pi \lambda}-1} \int_{0}^{2 \pi} d \Theta \int_{0}^{\infty} d \xi \omega^{2} \delta^{\prime}\left(\frac{\omega^{2}}{2} \xi+\frac{\omega^{2}}{2} \rho^{2}-\frac{1}{2} \omega^{2} a^{2}\right) \mathcal{G} \\
& =\frac{2 K}{\pi a^{2}} \frac{1}{e^{-i 2 \pi \lambda}-1}\left[\left.\delta\left(\frac{\omega^{2}}{2} \rho^{2}-\frac{1}{2} \omega^{2} a^{2}\right) \mathcal{G}\right|_{\xi=0}+\left.\frac{1}{\pi \omega^{2}} \int_{0}^{2 \pi} d \Theta \frac{d \mathcal{G}}{d \xi}\right|_{\xi=a^{2}-\rho^{2}}\right] .
\end{aligned}
$$

The second equality in (A15) follows after an integration by parts. This last equation is the same as Eq. (18).

\section{APPENDIX B}

In this Appendix we show that the RHS of Eq. (20) vanishes,

$$
\int_{0}^{2 \pi} d \Theta \frac{d G}{d \xi}=0
$$

if $\tilde{\psi}_{1}\left(x^{\prime}, y^{\prime}\right)+v_{z} \gamma^{2} \tilde{A}_{z}\left(x^{\prime}, y^{\prime}\right)$ is a harmonic polynomial. With $\tilde{A}_{z}$ as defined in the line below Eq. (17), $\tilde{\psi}_{1}$ as defined in Eq. (24), and $G$ as defined in (19), we have

$$
\frac{d \mathcal{G}}{d \xi}=i \lambda\left(\psi_{1, n}+v_{z} \gamma^{2} A_{z, n}\right) \int_{0}^{2 \pi} d \tau\left(\rho^{\prime}\right)^{n} \cos n \phi^{\prime} .
$$

Here $\rho^{\prime}$ and $\phi^{\prime}$ are given in terms of $x^{\prime}$ and $y^{\prime}$ by $x^{\prime}=\rho^{\prime} \cos \phi^{\prime}, y^{\prime}=\rho^{\prime} \sin \phi^{\prime}$, and in turn $x^{\prime}$ and $y^{\prime}$ are given by [see Eq. (A1)]

$$
\begin{aligned}
& x^{\prime}=x \cos \tau+\sqrt{\xi} \cos \Theta \sin \tau, \\
& y^{\prime}=y \cos \tau+\sqrt{\xi} \sin \Theta \sin \tau .
\end{aligned}
$$

If we define the function $\mathcal{P}_{n}(x, y, \xi)$ as

$$
\mathcal{P}_{n}(x, y, \xi)=\int_{0}^{2 \pi} d \Theta\left(\rho^{\prime}\right)^{n} \cos n \phi^{\prime},
$$

it is clear that (B1) is equivalent to

$$
\frac{d}{d \xi} \mathcal{P}_{n}(x, y, \xi)=0 \text {. }
$$

We will prove (B5) by showing that $\mathcal{P}_{n}(x, y, \xi)$ does not depend on $\xi$. We have

$$
\begin{aligned}
\mathcal{P}_{n}(x, y) & =\sum_{k=0}^{[n / 2]}(-1)^{k}\left(\begin{array}{c}
n \\
2 k
\end{array}\right) \int_{0}^{2 \pi} d \Theta\left(x^{\prime}\right)^{n-2 k}\left(y^{\prime}\right)^{2 k} \\
& =\sum_{s, p} c_{s, p}(\cos \tau)^{s+p}(\sin \tau)^{n-s-p}(\sqrt{\xi})^{n-s-p} x^{s} y^{p},
\end{aligned}
$$

with

$$
\begin{aligned}
c_{s, p}= & \sum_{k=[p / 2]}^{[(n-s) / 2]}(-1)^{k}\left(\begin{array}{c}
n \\
2 k
\end{array}\right)\left(\begin{array}{c}
n-2 k \\
s
\end{array}\right)\left(\begin{array}{c}
2 k \\
p
\end{array}\right) \\
& \times \int_{0}^{2 \pi} d \Theta(\cos \Theta)^{n-s-2 k}(\sin \Theta)^{2 k-p}
\end{aligned}
$$


The integral in $\Theta$ vanishes if either $n-s$ or $p$ are odd numbers. If both $n-s$ and $p$ are even numbers, the integral can be carried out using the formula

$$
\int_{0}^{2 \pi} d \Theta \cos ^{2 m} \Theta \sin ^{2 n} \Theta=\frac{\pi}{2^{m+n-1}} \frac{(2 m-1) ! !(2 n-1) ! !}{(m+n) !} .
$$

Therefore, we have

$$
\begin{aligned}
c_{s, p} & =\frac{2 \pi}{(\sqrt{2})^{n-s-p}} \sum_{k=p / 2}^{(n-s) / 2}(-1)^{k}\left(\begin{array}{c}
n \\
2 k
\end{array}\right)\left(\begin{array}{c}
n-2 k \\
s
\end{array}\right)\left(\begin{array}{c}
2 k \\
p
\end{array}\right) \frac{(n-s-2 k-1) ! !(2 k-p-1) ! !}{\left(\frac{n-s-p}{2}\right) !} \\
& =\frac{2}{(\sqrt{2})^{n-s-p}} \frac{1}{\left(\frac{n-s-p}{2}\right) !} \frac{1}{s ! p !} \sum_{k=p / 2}^{(n-s) / 2}(-1)^{k} \frac{1}{(n-s-2 k) ! !} \frac{1}{(2 k-p) ! !} .
\end{aligned}
$$

The coefficients $c_{s, p}$ vanish if $t=(n-s-p) \neq 0$ because

$$
\sum_{k=p / 2}^{(n-s) / 2}(-1)^{k} \frac{1}{(n-s-2 k) ! !} \frac{1}{(2 k-p) ! !}=\sum_{m=0}^{t}(-1)^{m} \frac{1}{(2 m) ! !} \frac{1}{(2 t-2 m) ! !}=\frac{t !}{2^{t}} \sum_{m=0}^{t}(-1)^{m}\left(\begin{array}{c}
t \\
m
\end{array}\right)=0 .
$$

On the other hand, if $(n-s-p)=0$ from Eq. (B6) we see the term proportional to the only surviving $c_{s, p}$ has no dependence on $\xi$. This ends the proof.

\section{APPENDIX C}

In Sec. IV we used the first-order solutions of the linearized Vlasov-Poisson equations to show that for a KV beam the effects of the $m$-harmonic component of a multipole error of order $n$ in the lattice are exactly compensated by the forces associated with the redistribution of the space charge caused by that lattice error if the tune has the values $\nu=m / n$. The purpose of this Appendix is to further illustrate this cancellation mechanism by using the envelope equations. For simplicity we consider the case of a round $\mathrm{KV}$ beam of unperturbed radius $a_{0}$ and with emittance $\varepsilon$ in an axially symmetric smooth channel with solenoidal-like errors. Let us then write the focusing function as $\omega_{0}^{2}\left[1+\alpha_{m} \cos \left(m \Omega_{0} z\right)\right]$, where $\alpha_{m}$ is the amplitude of the $m$ harmonic of the (small) solenoidal errors. The envelope equation including the perturbation reads

$$
a^{\prime \prime}+\omega_{0}^{2}\left(1+\alpha_{m} \cos m \Omega_{0} z\right) a-\frac{K}{a}-\frac{\varepsilon^{2}}{a^{3}}=0 .
$$

We linearize $(\mathrm{C} 1)$ after writing the envelope as $a=$ $a_{0}+u$ with $u / a_{0}$ being small; we have

$$
u^{\prime \prime}+p^{2} u=-\alpha_{m} \omega_{0}^{2} a_{0} \cos \left(m \Omega_{0} z\right)
$$

with

$$
p^{2}=\omega_{0}^{2}+\frac{K}{a_{0}^{2}}+3 \frac{\varepsilon^{2}}{a_{0}^{4}}=4 \omega^{2}+2 \frac{K}{a_{0}^{2}},
$$

and $\omega^{2}=\omega_{0}^{2}-K / a_{0}^{2}$ being, as usual, the depressed focusing function in the smooth approximation for the unperturbed beam. A solution of $(\mathrm{C} 2)$ is

$$
u=\frac{\alpha_{m} \omega_{0}^{2} a_{0}}{m^{2} \Omega_{0}^{2}-p^{2}} \cos \left(m \Omega_{0} z\right) .
$$

Now, let us consider the motion of an individual particle. The key point here is to take into account the variation of the beam radius due to the errors. The equation of motion reads

$$
x^{\prime \prime}+\omega_{0}^{2}\left[1+\alpha_{m} \cos \left(m \Omega_{0} z\right)\right] x-\frac{K}{\left(a_{0}+u\right)^{2}} x=0 .
$$

After linearization, using for $u$ the expression (C4), we have

$$
x^{\prime \prime}+\omega^{2} x=\left(\frac{2 K}{a_{0}^{2}} \frac{1}{p^{2}-m^{2} \Omega_{0}^{2}}-1\right) x \alpha_{m} \cos \left(m \Omega_{0} z\right) .
$$

With the help of (C3), the quantity in parentheses in the RHS of the equation above can be written as

$$
\left(\frac{2 K}{a_{0}^{2}} \frac{1}{p^{2}-m^{2} \Omega_{0}^{2}}-1\right)=\frac{m^{2} \Omega_{0}^{2}-4 \omega^{2}}{p^{2}-m^{2} \Omega_{0}^{2}},
$$

from which we can readily conclude that if $\omega / \Omega_{0}=\mathrm{m} / 2$ (i.e., if the tune is a half-integer) the RHS of Eq. (C6) vanishes and there is no net first-order perturbation on the motion of an individual particle.

[1] L. J. Laslett, in Proceedings of the Summer Study on Storage Rings, Brookhaven National Laboratory, 1963 (Brookhaven National Laboratory, Upton, NY, 1963), p. 324.

[2] R. Baartman, in Workshop on Space Charge Physics in High Intensity Hadron Rings, edited by A. U. Luccio and W. T. Weng, AIP Conf. Proc. No. 448 (AIP, New York, 1998).

[3] R. Cappi et al., in Proceedings of the Particle Accelerator Conference, Washington, DC, 1993 (IEEE, Piscataway, NJ, 1994), p. 3570.

[4] S. Machida, Nucl. Instrum. Methods Phys. Res., Sect. A 309, 43 (1991). 
[5] L. Smith, in Proceedings of the International Conference on High Energy Accelerators, Dubna, 1963 (AEC, Oak Ridge, TN, 1965), p. 1238.

[6] F. Sacherer, Ph.D. thesis, University of California, Berkeley, 1968; LNBL Report No. UCRL-18454, 1968.

[7] R. L. Gluckstern, in Proceedings of the Linac Conference, 1970 (Fermilab, Batavia, IL, 1970), p. 811.

[8] M. Reiser, Theory and Design of Charged Particle Beams (Wiley, New York, 1994).

[9] I. Hofmann, Phys. Rev. E 57, 4713 (1998).

[10] W. T. Weng, in Physics of Particle Accelerators, edited by Melvin Month and Margaret Deines, AIP Conf. Proc. No. 153 (AIP, New York, 1987), p. 348.
[11] This Hamiltonian has $z$, the longitudinal coordinate, as the independent variable. It is normalized in such a way that the canonical momenta $p_{x}$ and $p_{y}$ have the meaning of small angle deviations from the reference orbit.

[12] M. Reiser et al., in Proceedings of the 1999 Particle Accelerator Conference, New York (IEEE, Piscataway, NJ, 1999), p. 234.

[13] I. Hofmann, L. J. Laslett, S. Smith, and I. Haber, Part. Accel. 13, 145 (1983).

[14] R. L. Gluckstern, W. H. Cheng, and H. Y. Ye, Phys. Rev. Lett. 75, 2835 (1995).

[15] R. L. Gluckstern, Phys. Rev. Lett. 73, 1247 (1994). 